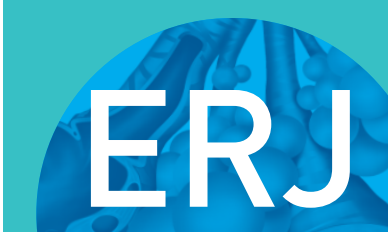

open research
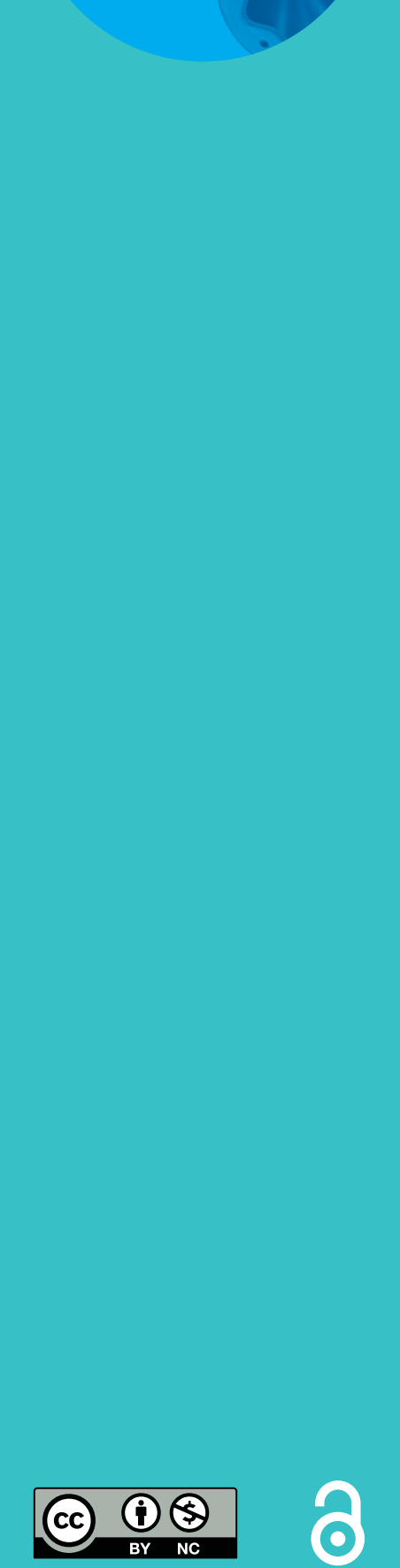

\title{
Transbronchial cryobiopsy: an effective tool in the diagnosis of lymphoproliferative disorders of the lung
}

\author{
Rita Bianchi ${ }^{1}$, Alessandra Dubini ${ }^{2}$, Silvia Asioli ${ }^{2}$, Claudia Ravaglia $^{3}$, \\ Sara Tomassetti ${ }^{3}$, Silvia Puglisi ${ }^{3}$, Sara Piciucchi $\mathbb{1}^{4}$, Christian Gurioli ${ }^{3}$, \\ Carlo Gurioli ${ }^{3}$, Roberto Fiocca ${ }^{1}$ and Venerino Poletti $i^{3,5}$
}

Affiliations: ${ }^{1}$ Dept of Pathology, Università di Genova, Ospedale Policlinico San Martino, Genoa, Italy. ${ }^{2}$ Dept of Pathology, Ospedale GB Morgagni, Forli, Italy. ${ }^{3}$ Dept of Diseases of the Thorax, Ospedale GB Morgagni, Forli, Italy. ${ }^{4}$ Dept of Radiology, Ospedale GB Morgagni, Forli, Italy. ${ }^{5}$ Dept of Respiratory Diseases and Allergy, Aarhus University Hospital, Aarhus, Denmark.

Correspondence: Rita Bianchi, Anatomia Patologica, Università di Genova and IRCCS Ospedale Policlinico San Martino, Largo Rosanna Benzi 10, 16132, Genova (GE), Italy. E-mail: bianchirita22agmail.com.

\section{ABSTRACT}

Introduction: Malignant lymphoproliferative disorders are rarely observed in the lung and, considering their clinical and radiological heterogeneity, diagnosis is often difficult and may require invasive methods. Transbronchial cryobiopsy has been confirmed as a new tool in the diagnosis of interstitial lung diseases, given its fewer risks and costs compared to surgical approach. This study is aimed at assessing the effectiveness of cryobiopsy in the diagnosis of lymphoproliferative disorders.

Materials and methods: Among 970 consecutive cryobiopsies, performed between January 2011 and June 2018 at Morgagni Hospital of Forlì, Italy, 13 cases of lymphoproliferative disorders were collected.

Results: In 12 out of 13 cases a precise pathological diagnosis could be reached with the support of immunohistochemistry (IHC) and molecular ancillary studies. In the only case in which cryobiopsy did not lead to a definitive diagnosis, the subsequent surgical biopsy also did not help to clarify the diagnosis. Severe bleeding or pneumothorax did not occur in any case. On average, five biopsies were obtained per case, with a mean total area of $1161 \mathrm{~mm}^{2}$, and only 5 out of 65 specimens were inadequate for diagnosis. Instant freezing did not produce tissue artefacts nor did it affect IHC and molecular tests. In all cases the amount of available tissue was sufficient for all ancillary studies.

Conclusions: Transbronchial lung cryobiopsy is safe and effective for diagnosis in patients with suspected pulmonary involvement by lymphoproliferative disorders and it should therefore be considered a valid alternative to surgical biopsy in such cases.

@ERSpublications

Cryobiopsy is an effective method in the diagnosis of lymphoproliferative disorders. Transbronchial cryobiopsy allows immunohistochemical and molecular analysis. No severe bleeding or pneumothorax were observed in this study. https://bit.ly/2 $\mathrm{CsHCx} 9$

Cite this article as: Bianchi R, Dubini A, Asioli S, et al. Transbronchial cryobiopsy: an effective tool in the diagnosis of lymphoproliferative disorders of the lung. ERJ Open Res 2020; 6: 00260-2019 [https://doi.org/10.1183/23120541.00260-2019]. 


\section{Introduction}

Malignant lymphoproliferative disorders are rarely observed as primary lesions in the lung, accounting for $<0.5 \%$ of all primary pulmonary malignancies $[1,2]$. These are usually defined as a clonal lymphoid proliferation affecting one or both lungs (parenchyma and/or bronchi) in patients with no detectable extrapulmonary involvement at the time of diagnosis or during the subsequent 3 months [2]. This definition, however, is not precise because indolent extranodal lymphoma may present clinically and radiologically as primary pulmonary lesions and aggressive lymphoid tumours may initially manifest as disorders mainly involving the respiratory tract. Primary pulmonary lymphomas should, therefore, be defined as lymphoid neoplasms which become manifest as respiratory diseases $[3,4]$.

The World Health Organization (WHO) classification of tumours of the lung [2] categorises primary pulmonary lymphomas into: B-cell primary pulmonary non-Hodgkin lymphomas including marginal zone B-cell lymphoma of mucosa-associated lymphoid tissue (MALT) type, primary pulmonary diffuse large B-cell lymphoma and lymphomatoid granulomatosis. Nevertheless, the lung may be the primary site of presentation of most types of usually lymph-node-based lymphomas [3].

Secondary involvement of the lung, on the other hand, is relatively common in patients with malignant lymphoma. It may be caused by direct invasion from involved mediastinal lymph nodes or diffusion via the blood stream or lymphatics [5].

The clinical presentation of malignant lymphoproliferative diseases of the lung may differ on the basis of the aggressiveness of the disease. It can present as an asymptomatic solitary mass on routine chest radiography in "low-grade" diseases, or with symptoms like cough, dyspnoea, chest pain, haemoptysis and systemic "B" symptoms in "high-grade" lymphomas [5]. The presence of autoimmune disorders and the related immunosuppressive therapies are considered possible risk factors.

Imaging studies show either unilateral or bilateral disease, with isolated or multiple opacities; diffuse infiltration, reticular-nodular shadowing and pleural effusions have been also described.

Historically, a surgical biopsy was the usual diagnostic approach but, with advances in high-resolution computed tomography allowing better clinical-pathological correlation (figure 1) and the advent of molecular techniques, bronchoscopic transbronchial biopsies (TBB), bronchoalveolar lavage [6] and fine-needle aspiration have been successfully used for diagnosis [5, 7-9]. These approaches, however, are diagnostic in only $30-50 \%$ of cases [2-4] and this is mainly due to the tiny samples obtained.

Transbronchial cryobiopsy (cryo-TBB) is a relatively new method that allows to obtain larger samples of lung tissue, without crushing artefacts, and increases the diagnostic yield of traditional transbronchial biopsies in diffuse parenchymal lung diseases [10-14].

We reviewed all transbronchial cryobiopsies performed at G.B. Morgagni Hospital in Forlì (Italy) between January 2011 and June 2018 and isolated those cases with a biopsy diagnosis of lymphoproliferative disorder (both primary and secondary lung involvements). The aim of the study was to assess the effectiveness of cryo-TBB in the diagnosis of these rare lung malignancies.

\section{Materials and methods}

The retrospective evaluation of a continuous series of 970 cryo-TBB performed at G.B. Morgagni Hospital of Forlì (Italy) between January 2011 and June 2018 identified a total of 13 cases of malignant

FIGURE 1 Computed tomography scan of case no. 1: consolidation area in the upper right lobe, with a partial component of ground glass and surrounding thickening of intra and interlobular septa.

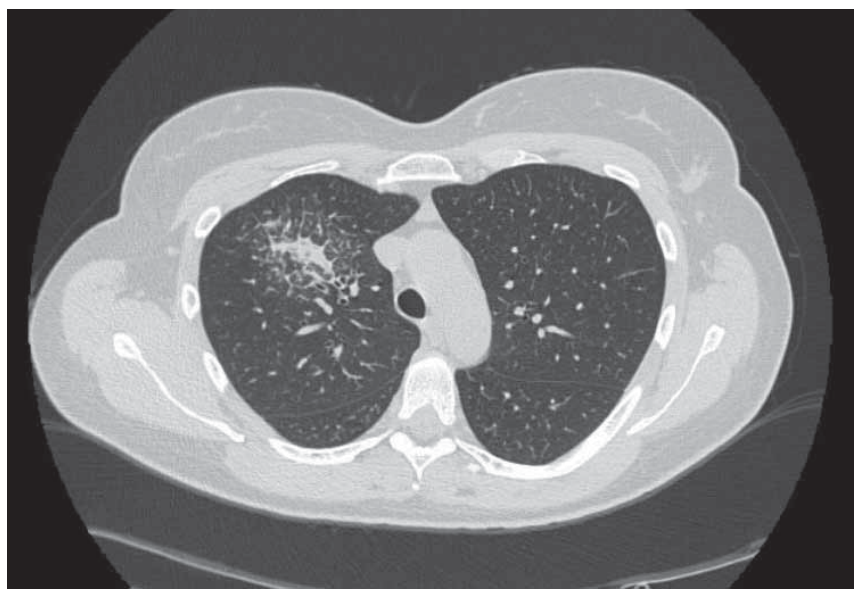


lymphoproliferative disorders (both primary and secondary involvements of the lung). The search was not performed on the whole histological database, but restricted to the full series of cryo-TBB, for which both clinical-radiological picture and final histological diagnosis were known. As far as known, we have no evidence that any case submitted to cryo-TBB received a diagnosis of lymphoproliferative disorder during subsequent follow-up, excluding the 13 cases included in the present report. For these reasons, we are confident that no lymphoproliferative disorder was missed.

The study was an anonymised retrospective review of cases and neither patient consent nor ethical approval was sought. All patients attending the Morgagni-Pierantoni Hospital were informed about the use of patient data for continuing research at the hospital.

Cryo-TBBs were carried out as already described [15]. Briefly, all patients were deeply sedated with propofol and remiphentanil and intubated with a 14-mm diameter rigid tracheoscope (Storz, Stutgard, Germany). All patients remained in spontaneous ventilation. A Fogarty balloon (4 French) was preventively introduced up to the segment to biopsy to control eventual haemorrhages. Bleeding was defined as "mild" if requiring just endoscopic aspiration, "moderate" if requiring further endoscopic procedures (bronchial occlusion and/or instillation of ice-cold saline), and "severe" if requiring surgical interventions, transfusions and/or admission to intensive care unit for haemodynamic or respiratory instability. Biopsies were obtained using a 2.4 cryoprobe with a freezing time of $5-6 \mathrm{~s}$ and under fluoroscopic control. In cases with alveolar consolidation, the target was also identified using a radial (20 $\mathrm{mHtz})$ endobronchial ultrasound probe.

Only two patients underwent an endobronchial ultrasound-guided transbronchial needle aspiration (EBUS-TBNA) because of lymph node enlargement: in patient no. 9, a subcarinal lymph node was examined, while in patient $\mathrm{n}^{\circ} 10$ the selected lymph node was paratracheal.

All samples obtained with cryoprobes were fixed in formalin, routinely processed and embedded in paraffin and microtome cut, obtaining at least three levels of section stained with haematoxylin \& eosin (figure 2). All specimens were measured with a digital microscope (Leika DMD108) in order to evaluate the dimensional adequacy of biopsies.

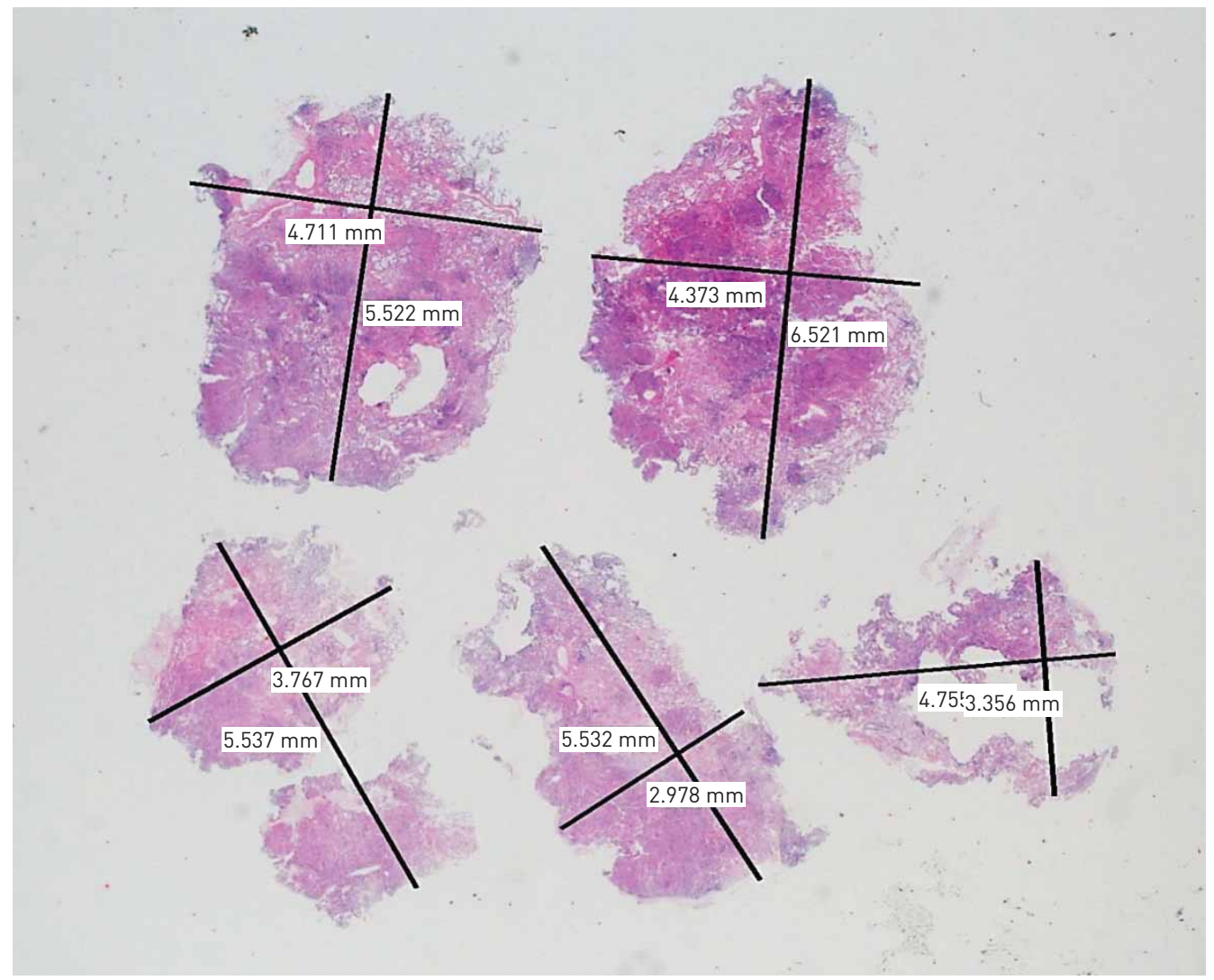

FIGURE 2 Haematoxylin \& eosin $\times 20$ : low magnification of case no. 2, showing the number of samples and their size. 
Immunohistochemistry (IHC) for B- and T-lymphocytes was performed in all cases with a combination of different antibodies for every case, on the basis of morphology, in order to identify the phenotype of lesions. The following antibodies were used: CD20, CD3, CD5, CD8, CD10, CD21, CD23, CD15, CD30, bcl2, bcl6, ki67, IRF4/MUM1, Cyclin-D1, PAX5, ALK, OCT2, LCA and BOB1, all provided by Ventana Medical Systems, Inc (Oro Valley, Arizona, USA).

In situ hybridisation (ISH) was performed on some samples. In particular, translocation of BIRC3/MALT1 $(\mathrm{t}(11: 18)(\mathrm{q} 21: \mathrm{q} 21)$, the most frequent genetic aberration in MALT lymphoma [2]) was evaluated with fluorescence in situ hybridisation (FISH) method in five cases (no. 1, 6, 8, 12 and 13). Rearrangement of IgH (which can provide evidence of clonal expansion of heavy chains of immunoglobulins [2]) was evaluated with the FISH method in three cases (no. 5, 6 and 13). Rearrangement of MYC gene (which can be identified in diffuse large B-cell lymphoma [2]) was evaluated only for case no. 11. Epstein-Barr virus (EBV) expression, which can be involved in diffuse large B-cell lymphoma and lymphoid granulomatosis, was identified with ISH in six cases [2, 3].

\section{Results}

\section{Clinical aspects}

Clinical profiles, available relevant laboratory and pulmonary function data, bronchoalveolar lavage (BAL) and CT scan findings are reported in table 1.

All patients showed similar clinical symptoms: they presented with light or recurring fever and cough. Three patients (no. 2, 9 and 12) had a former diagnosis of lymphoproliferative disease, and so cryo-TBBs were performed in order to confirm the hypothesis of lung involvement in disease's relapse. Five patients had a previous diagnosis of autoimmune disease, infections or monoclonal gammopathies of uncertain significance.

Pulmonary functional tests generally showed a mild restrictive deficit or normal findings. The majority of cases (9 out of 13) showed lymphocytosis on BAL and 7 of them had a lymphocyte differential count $>25 \%$, which is suggestive of granulomatous lung disease or lymphoproliferative lung disorder [16].

Radiological patterns were also similar in all cases, showing interstitial thickening, ground-glass areas and/ or nodules, mostly bilateral, mimicking diffuse interstitial lung disease (more often acute, such as organising pneumonia or diffuse alveolar damage). After cryo-TBBs no severe bleeding or pneumothorax was observed (only case no. 11 presented moderate bleeding after the procedure).

\section{Histology}

The size of biopsies obtained with a cryoprobe are described in table 2: the mean number of specimens for case was 5, with a mean total area of $1161 \mathrm{~mm}^{2}$ and a median value of $81.01 \mathrm{~mm}^{2}$.

Histologically, 60 out of 65 specimens were adequate for diagnosis; inadequate samples contained only bronchiolar walls, fibrin, pleura or striated muscle cells (table 2). In all cases an infiltrate of lymphoid cells was seen, with variable extension and distribution, but sufficient to hypothesise a lymphoproliferative disease.

EBUS-TBNAs from patients no. 9 and 10 showed no evidence of lymphoproliferative disorder.

Based on histology, IHC and subsequent molecular studies, integrated with clinical and radiological background, nine primary lung malignant lymphoproliferative disorders and three secondary localisations from a previously known lymphoma (table 1) were diagnosed.

- In one case (no. 13) the histological and immunohistochemical findings were inconclusive and the case was reported as "dense infiltrate of atypical lymphocytes not sufficient for the diagnosis of lymphoma". This patient subsequently underwent a surgical lung biopsy but, despite the availability of a larger tissue sample, a specific diagnosis could not be reached. In fact, both the cryobiopsy and the surgical showed a dense atypical lymphocytic infiltrate, with a mixture of B-cells and T-cells, and even if a restriction for the heavy chain of immunoglobulin was found, histological and immunohistochemical findings were inconclusive.

- Three cases (no. 1, 6 and 8) were diagnosed as MALT lymphomas. All three cases showed a distribution surrounding small bronchioles and pulmonary arteries and two cases were located beneath the visceral pleura and in perilobular septa. The same two cases also presented typical lymphoepithelial lesions. Cases no. 6 and 8 showed a restricted expression of immunoglobulins. Cases no. 1 and 8 resulted translocated with FISH assessment of BIRC3/MALT1. Case no. 6 showed rearrangement of IgH chains.

- Two cases (no. 3 and 4) were diagnosed as intravascular large B-cell lymphoma [17], showing intravascular distribution of large atypical lymphoid cells, without other evident parenchymal alterations. Atypical lymphoid cells were found in both capillaries and small arterioles (case no. 3) or only in capillaries (case no. 4). Both cases have been previously published [10]. 
TABLE 1 Clinical and radiological findings

\section{Case no., sex, age yrs}

\section{Laboratory findings and functional pulmonary data}

1, female, 38 Former smoker. Dry cough and light fever. No relevant auscultatory sounds.

Suspect for lymphoproliferative disorder.

2, female, 41

3, male, 68

4, male, 57

5 , female, 70

Hashimoto thyroiditis and Sjögren syndrome. Light fever. MALT lymphoma? other?

6, female, 63

7, female, $31 \quad$ Nonsmoker; office worker. Former mononucleosis; asthma. Light fever, sweating, dry cough and pain at right shoulder.
$\mathrm{S}_{\mathrm{aO}_{2}} 99 \%$.

FVC $102 \%$

$\mathrm{FEV}_{1} 99 \%$

$D_{\mathrm{LCO}} 49 \%$

Lymphopenia (total lymphocytes: $0.4210^{9}$ ).

FVC $100 \%$

FEV 1 100\%

$D_{\text {LCO }} 52 \%$

CRP 169; LDH 975.

Monoclonal $\lg G \lambda$

component on protein electrophoresis.

FVC $89 \%$

$\mathrm{FEV}_{1} 91 \%$

$D_{\text {LCO }} 53 \%$ (mild restrictive deficit)

Pancytopenia; LDH 3194. Monoclonal IgG $\kappa$ component on protein electrophoresis.

Pulmonary hypertension:

PAP $40 \mathrm{mmHg}$. Negative hypersensitivity specific antigens.

FVC 107\% $\mathrm{FEV}_{1} 109 \%$ $D_{\text {LCo }} 42 \%$

No significant alterations. FVC $81 \%$ FEV $181 \%$ $D_{\text {LCo }} 59 \%$

Specific antibodies for polymyositis: ku ++, Ro-52

+++ ; high CPK and myoglobin.

$P_{\mathrm{aO}_{2}}: 82.9 \mathrm{mmHg}, P_{\mathrm{aCO}_{2}}$ :

$42.5 \mathrm{mmHg}, \mathrm{pH}: 7.39 \mathrm{U}$.

Mild lymphopenia. Two monoclonal IgG

components lone $\kappa$ and one $\lambda$ ) on protein electrophoresis.

FVC 105\%

FEV1 93\%

DLCO 55\%

CRP $60.9 \mathrm{mg}$.

$\mathrm{L}^{-1}$; LDH 290.

FVC $101 \%$

$\mathrm{FEV}_{1} 91 \%$

$D_{\text {LCO }} 78 \%$
BAL

HRCT
Total cellularity: $40 \times 10^{6} \cdot \mathrm{L}^{-1}, \mathrm{~N} 6.0 \%$ E $0.0 \%, L 8.0 \%, M$ $86.0 \%$

Total cellularity: $630 \times 10^{6} \cdot \mathrm{L}^{-1}, \mathrm{~N} 0 \%$, E 3.0\%, L 77.0\%, M $20.0 \%$

Total cellularity: $270 \times 10^{6} \cdot L^{-1}, N$ $4.0 \%, \mathrm{E} 0.0 \%, \mathrm{~L}$ $26.0 \%, M 70.0 \%$

Total cellularity: $210 \times 10^{6} \cdot \mathrm{L}^{-1}, \mathrm{~N}$ $2.0 \%, \mathrm{E} 0.0 \%$, L $8.0 \%, \mathrm{M} 90.0 \%$

Total cellularity: $210 \times 10^{6} \cdot L^{-1}, N$ $3.0 \%$, E $0.0 \%$, L $30.0 \%$, M $67.0 \%$ Total cellularity: $200 \times 10^{6} \cdot \mathrm{L}^{-1}$ N $18 \%$, E $1 \%$, L $22 \%, M 5 \%$

Not available

Opacity at superior right lobe on CT scans and diffuse bone, lung, splenic and lymph nodes uptakes on PET.
Nodular opacities at superior lobe of right lung; multiple bilateral perilymphatic nodules, with ground-glass areas and thickening of septa.

Bilateral lung opacities, predominantly perivascular, mostly at inferior lobes, with areas of consolidation.

Diffuse density increasing and minimal consolidations in dorsal regions, mostly at inferior lobes. Suspect for DAD or OP.

Ground glass with expiratory air trapping and mediastinal adenopathy.

Suspect for intrinsic allergic alveolitis.

Parenchymal bilateral consolidations, with thickening of interstitial septa lthose nodules

were also PET-positive). Interlobular reticular septal thickening and ground glass, honeycombing: suspect of fibrotic evolution of former interstitial pathologies. 
TABLE 1 Continued

\section{Case no. sex, age yrs \\ Recent symptoms and suspected diagnosis}

9, female, 78

10, male, 35

11 , male, 40

12 , male, 60

13, female, 45 Nonsmoker. Went to ER for dry cough and thoracic pain; dyspnoea without fever.

Suspect for lymphoma.

\section{Laboratory findings and functional pulmonary data}

Monoclonal expression of $\lg \mathrm{G} \kappa$ light chains on BAL. FVC $100 \%$ $\mathrm{FEV}_{1} 85 \%$ $D_{\text {LCO }} 88 \%$

Mild lymphopenia. LDH 316; CRP 67.3.

Monoclonal $\lg G \mathrm{~K}$

component on protein electrophoresis. FVC 101\% FEV 1 102\% $D_{\text {LCo }} 76 \%$ Anaemia.

LDH 112; CRP 73.4. FVC $100 \%$ FEV $101 \%$ $D_{\text {LCo }} 73 \%$ Anaemia. Peripheral blood: total $\mathrm{CD}^{+}$lymphocytes: $77 \%$, with a CD4/CD8 ratio of 0.18 .

Functional pulmonary data not available Anaemia. FVC 103\% FEV 1 105\% $D_{\text {LCO }} 88 \%$

Anaemia. RCP 15. FVC $88 \%$ $\mathrm{FEV}_{1} 95 \%$ $D_{\text {LCo }} 60 \%$
BAL

HRCT
Total cellularity: $510 \times 10^{6} \cdot \mathrm{L}^{-1}$, N $33.0 \%$, E $2.0 \%$, L $55.0 \%, \mathrm{M} 10.0 \%$

Total cellularity: $90 \times 10^{6} \cdot \mathrm{L}^{-1}$

N $60.0 \%$, E $0.0 \%$, L $10.0 \%$, M $30.0 \%$
Bilateral lung nodules, persistent after antibiotics therapy (with ground-glass areas and interstitial thickening).

Lung opacities and mediastinal, abdominal and groin lymphadenopathy on CT-PET.
Total cellularity: $3720 \times 10^{6} \cdot L^{-1}$, N 6.0\%, E $0.0 \%, \mathrm{~L}$
Hilar and para-hilar parenchymal opacities with mediastinal adenopathy. $94.0 \%$

Total cellularity: $680 \times 10^{6} \cdot \mathrm{L}^{-1}$

N $25.0 \%$, E $0.0 \%$, L $50.0 \%$, M $25.0 \%$

Total cellularity: $80 \times 10^{6} \cdot \mathrm{L}^{-1}$ N $3.0 \%, E$ E $0.0 \%$, L $17.0 \%, M 80.0 \%$

Total cellularity: $330 \times 10^{6} \cdot L^{-1}$ N $3.0 \%$, E $0.0 \%, L$ $35.0 \%, M 60.0 \%$
Multiple solid lung nodules, bilateral but predominant on the right.

Bi-basal nodular areas, ground-glass areas, micronodular perilymphatic aspect, minimum pleuropericardic effusion. Splenomegaly.

Opacities with ground-glass halo, mediastinal and subdiaphragmatic adenopathy, splenomegaly with multiple hypodense lesions.

Lung function values are presented as \% predicted. HRCT: high-resolution computed tomography; $S_{a_{2}}$ : oxygen saturation of arterial blood: FVC: forced vital capacity; $\mathrm{FEV}_{1}$ : forced expiratory volume in $1 \mathrm{~s} ; \mathrm{D}_{\mathrm{Lco}}$ : diffusing capacity of the lung for carbon monoxide; N: neutrophils; E: eosinophils; L: lymphocytes; M: macrophages; EBV: Epstein-Barr virus; CRP: C-reactive protein; LDH: lactate dehydrogenase; MGUS: monoclonal gammopathy of unknown significance; HP: hypersensitivity pneumonia; PAP: pulmonary arterial pressure; MALT: mucosa-associated lymphoid tissue; DAD: diffuse alveolar damage; OP: organising pneumonia; NSIP: nonspecific interstitial pneumonia; CPK: creatine phosphokinase; $P_{\mathrm{aO}_{2}}$ : partial pressure of oxygen in arterial blood; $P_{\mathrm{aco}}$ : partial pressure of carbon dioxide in arterial blood; CT: computed tomography; PET: positron emission tomography; BAL: bronchoalveolar lavage; ER: emergency room.

- Two cases (no. 7 and 10) were diagnosed as classical Hodgkin lymphomas, nodular sclerosis, in a cellular phase variant. In both cases the infiltrate was very dense, with complete effacement of lung architecture. Only in case no. 7 did the infiltrate prevalently surround a recognisable bronchiolar wall. IHC investigations confirmed CD15 and CD30 expression in Reed-Sternberg cells (see figures 3 and 4).

- Two cases (no. 9 and 11) were diagnosed as diffuse large B-cell lymphoma (DLBCL). Case no. 9 had a previous diagnosis of DLBCL. Both cases showed a dense infiltrate of large blastic-like cells with destruction of normal lung architecture. Case no. 9 presented a distribution along peribronchovascular bundles, also with some lymphoepithelial complexes. Case no. 11 showed diffuse coagulative necrosis, with "ghost cells" and viable cells percolating along few blood vessels.

- One case (no. 12) was diagnosed as lymphoplasmacytic lymphoma, in a patient with a previous diagnosis of lymphoplasmocytic lymphoma/Walderström's macroglobulinaemia on bone marrow biopsy. The lymphoid infiltrate was located around pulmonary veins and in bronchovascular bundles. 
TABLE 2 Histological features

\begin{tabular}{|c|c|c|c|c|c|}
\hline Case no. & $\begin{array}{l}\text { No. } \\
\text { specimens }\end{array}$ & Not adequate & Total size & Distribution & Histological diagnosis \\
\hline 1 & 5 & - & $40.60 \mathrm{~mm}^{2}$ & Small lymphoid cells around blood vessels & MALT lymphoma \\
\hline 3 & 4 & - & $76.00 \mathrm{~mm}^{2}$ & $\begin{array}{c}\text { Large lymphoid elements in capillaries and } \\
\text { arterioles }\end{array}$ & intravascular NHL \\
\hline 6 & 4 & - & $81.01 \mathrm{~mm}^{2}$ & $\begin{array}{c}\text { Small lymphoid cells and plasma cells around } \\
\text { blood vessel and along lymphatic routes } \\
\text { (paraseptal and subpleural) }\end{array}$ & MALT lymphoma \\
\hline 7 & 7 & $\begin{array}{l}\text { 1: bronchial } \\
\text { wall }\end{array}$ & $73.20 \mathrm{~mm}^{2}$ & $\begin{array}{l}\text { Hodgkin cells and lymphoid infiltrate around } \\
\text { bronchiolar walls and vessels }\end{array}$ & $\mathrm{HL}$ \\
\hline 10 & 4 & - & $78.92 \mathrm{~mm}^{2}$ & Hodgkin cells and lymphoid infiltrate & $\mathrm{HL}$ \\
\hline 11 & 13 & - & $417.70 \mathrm{~mm}^{2}$ & $\begin{array}{l}\text { Large atypical lymphoid cells around blood } \\
\text { vessels. Coagulative necrosis }\end{array}$ & Diffuse large B-cell lymphoma \\
\hline 12 & 9 & 2: fibrin & $51.55 \mathrm{~mm}^{2}$ & $\begin{array}{c}\text { Small lymphoid cells around bronchovascular } \\
\text { bundles and blood vessels }\end{array}$ & Lymphoplasmocytic lymphoma \\
\hline 13 & 4 & $\begin{array}{l}\text { 2: pleura and } \\
\text { muscle }\end{array}$ & $235.11 \mathrm{~mm}^{2}$ & $\begin{array}{c}\text { Dense infiltration of small lymphoid cells in } \\
\text { subpleural area, resulted a mixture of B and } \\
\text { T-lymphoid cells }\end{array}$ & $\begin{array}{l}\text { Cryo-TBB and VATS: atypical } \\
\text { lymphoproliferative process }\end{array}$ \\
\hline
\end{tabular}

MALT: mucosa-associated lymphoid tissue; PTCL, NOS: peripheral T-cell lymphoma, not otherwise specified; NHL: non-Hodgkin lymphoma; LYG: lymphomatoid granulomatosis; EBV: Epstein-Barr virus; HL: Hodgkin lymphoma; cryo-TBB: transbronchial cryobiopsy; VATS: video-assisted thoracoscopic surgery.

- One case (no. 5) was diagnosed as a grade 2 lymphomatoid granulomatosis. The lymphoid infiltrate was evident around pulmonary arteries and also affected the walls of these vessels. Multifocal necrosis, with cellular debris, was also seen. EBER (Epstein-Barr encoding region) evaluation with the ISH method resulted positive: up to 12 per high power field of large blastic-appearing CD20-positive cells showed nuclear staining for EBV. This feature permitted the classification as a grade 2 lymphomatoid granulomatosis. Neoplastic cells showed a rearrangement of IgH, evaluated with FISH.

- Case no. 2 was diagnosed as lung involvement by T-cell lymphoma, not otherwise specified, of which the patient had a previous diagnosis. The infiltration was distributed around blood vessels and in paraseptal zones.

FIGURE 3 Haematoxylin \& eosin $\times 100$ : case no. 7. A diffuse and mixed infiltration is present, with eosinophils, lymphocytes and large blastic elements, consistent with Hodgkin cells.

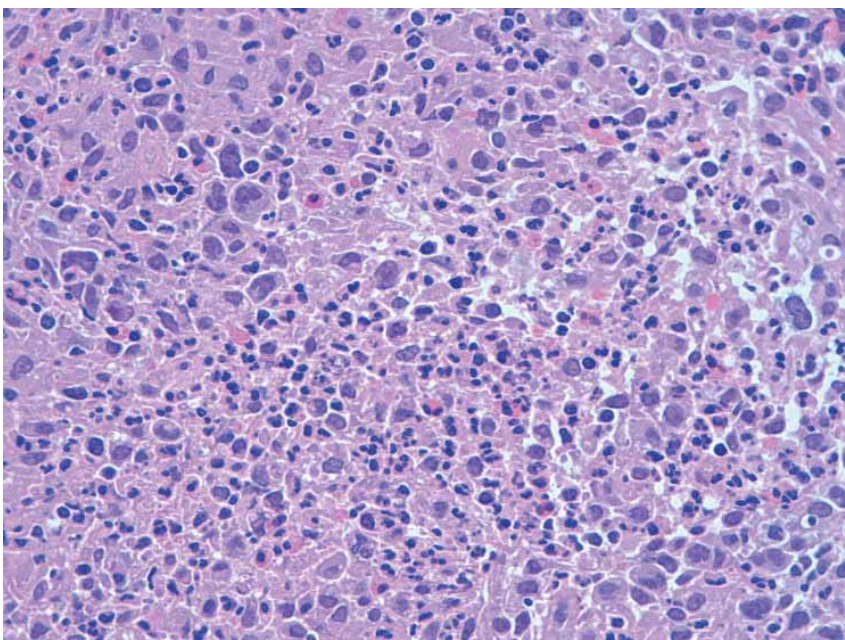




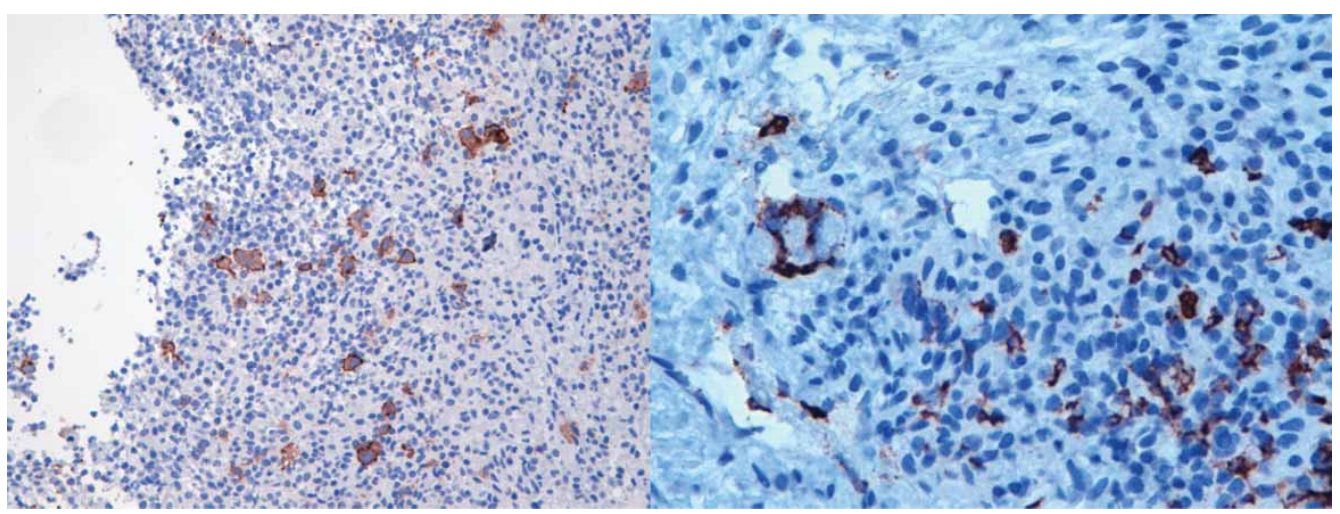

FIGURE 4 Immunohistochemical stain for CD30 (left) and CD15 (right), $\times 200$ : case no. 7. The large elements are strongly positive both for CD30 and CD15, confirming the diagnosis of classic Hodgkin lymphoma.

Histochemical stains (Ziehl-Neelsen, Grocott and PAS) were performed in cases no. 2, 5 and 11 in order to exclude the presence of mycobacteria and fungi and were negative. Cytomegalovirus IHC was performed in cases no. 2 and 11 and was negative.

Three cases presented scattered intra-alveolar macrophages (no. 1, 2, 6). Two cases showed intra-alveolar fibrin (no. 11 and 12) and two cases, focal ossification (no. 3 and 8). In all other cases the adjacent parenchyma showed no relevant lesions.

\section{Discussion}

The diagnosis of lymphoproliferative lung disease is often difficult due to the heterogeneity of the disease. Furthermore, diagnosis can be problematic if solely based on microscopy and therefore requires the use of ancillary techniques, including immunophenotypic characterisation by IHC and/or ISH, flow cytometry and molecular testing $[3,5]$.

The description of cases of lymphoproliferative diseases diagnosed on conventional transbronchial biopsy is anecdotal and in the literature there are no series of cases that demonstrate the efficiency of transbronchial biopsy using flexible forceps. Moreover, traditional transbronchial biopsies provide an insufficient amount of tissue for complete $\mathrm{IHC} /$ molecular analysis and samples are often hampered by crush artefacts [18]. Therefore, surgical lung biopsy in video thoracoscopy is considered the gold standard to obtain adequate tissue in interstitial lung diseases $[14,18]$. However, many patients with interstitial lung diseases have comorbidities and clinical conditions which increase complication rates.

Cryo-TBB was introduced as a new technique in the diagnosis of interstitial lung diseases [9], improving the diagnostic yield of conventional transbronchial biopsy by providing a larger amount of tissue for pathological diagnosis, while having a minor complication rate.

The results of this study with cryo-TBBs show that such a diagnostic approach is safe even in the context of lymphoproliferative disorders as cryo-TBB is an appropriate tool for the evaluation of the nature, distribution and extension of a lymphoid infiltrate in the lung. Normal pleural-parenchymal structures are recognisable and well preserved and instant freezing does not produce significant artefacts [14, 18] nor does it affect immunoreactivity [3] or molecular tests. Moreover, IHC and molecular tests can be easily performed given the sufficient amount of tissue available.

This is, to our knowledge, the largest series of lymphoproliferative lung diseases diagnosed on cryoprobe specimens as only two previous case reports (both DLBCL) are present in the literature $[18,19]$.

A precise definition of the lymphoid infiltrate was obtained in 12 out of the selected 13 cases while only 1 patient of this series (case no. 13) with an inconclusive cryobiopsy underwent a subsequent surgical lung biopsy that, despite the availability of a larger tissue sample, did not allow to reach a specific diagnosis.

Transbronchial lung cryobiopsy should therefore be considered a valid alternative to surgical lung biopsy also in patients with suspected pulmonary involvement by lymphoproliferative disorders $[1,3]$. It permits the acquisition of sufficient and suitable tissue samples, causing far fewer complications than surgical biopsy, making it feasible in patients with comorbidities, which would otherwise not tolerate surgical procedures $[10,14,18]$.

Author contributions: V. Poletti, R. Bianchi and R. Fiocca conceptualised the study, performed the formal analysis, acquired funding, wrote the original draft of the manuscript, and reviewed and edited the final version. A. Dubini, 
S. Asioli, S. Tomassetti, S. Puglisi, C. Ravaglia, S. Piciucchi, C. Gurioli and C. Gurioli curated and validated the data, and reviewed and edited the manuscript.

Conflict of interest: None declared.

\section{References}

1 Chilosi M, Zinzani PL, Poletti V. Lymphoproliferative lung disorders. Semin Respir Crit Care Med 2005; 26: 490-501.

2 Travis WD, Brambilla E, Burke AP, et al. WHO classification of tumors of the lung, pleura, thymus and heart. 4th Edn. Lyon, IRAC, 2015.

3 Poletti V, Ravaglia C, Tomassetti S, et al. Lymphoproliferative lung disorders: clinicopathological aspects. Eur Respir Rev 2013; 22: 427-436.

4 Poletti V, Casoni GL, Piciucchi S, et al. Lymphoproliferative lung disorders. In: Cottin V, Cordier JF, Richeldi L, eds. Orphan Lung Diseases. London, Springer Verlag, 2015; pp. 493-515.

5 Nicholson AG. Lymphocytic interstitial pneumonia and other lymphoproliferative disorders in the lung. Semin Respir Crit Care Med 2001; 22: 409-422.

6 Poletti V, Romagna M, Gasponi A, et al. Bronchoalveolar lavage in the diagnosis of low-grade, MALT type, B-cell lymphoma in the lung. Monaldi Arch Chest Dis 1995; 50: 191-194.

7 Takamura K, Nasuhara Y, Mishima T, et al. Intravascular lymphomatosis diagnosed by transbronchial lung biopsy. Eur Respir J 1997; 10: 955-957.

8 Kaku N, Seki M, Doi S, et al. A case of intravascular large B-cell lymphoma (IVLBCL) with no abnormal findings on chest computed tomography diagnosed by random transbronchial lung biopsy. Intern Med 2010; 49: 2697-2701.

9 Nakazato T, Sanada Y, Mihara A, et al. PET negative pulmonary intravascular large B cell lymphoma diagnosed by a random transbronchial lung biopsy. Ann Hematol 2012; 91: 811-812.

10 Poletti V, Gurioli C, Piciucchi S, et al. Intravascular large B cell lymphoma presenting in the lung: the diagnostic value of transbronchial cryobiopsy. Sarcoidosis Vasc Diffuse Lung Dis 2015; 31: 354-358.

11 Babiak A, Hetzel J, Krishna G, et al. Transbronchial cryobiopsy: a new tool for lung biopsies. Respiration 2009; 78 203-208.

12 Casoni GL, Tomassetti S, Cavazza A, et al. Transbronchial lung cryobiopsy in the diagnosis of fibrotic interstitial lung diseases. PLoS One 2014; 9: e86716.

13 Poletti V, Casoni GL, Gurioli C, et al. Lung cryobiopsies: a paradigm shift in diagnostic bronchoscopy? Respirology 2014; 19: 645-654.

14 Colby TV, Tomassetti S, Cavazza A, et al. Transbronchial cryobiopsy in diffuse lung disease: Update for the pathologist. Arch Pathol Lab Med 2017; 141: 891-900.

15 Colella S, Haentschel M, Shah P, et al. Transbronchial lung cryobiopsy in interstitial lung diseases: best practice. Respiration 2018; 95: 383-391.

16 Meyer KC, Raghu G, Baughman RP, et al. An official American Thoracic Society clinical practice guideline: the clinical utility of bronchoalveolar lavage cellular analysis in interstitial lung disease. Am J Respir Crit Med 2012; 185: 1004-1014.

17 Walls JG, Hong YG, Cox JE, et al. Pulmonary intravascular lymphomatosis: presentation with dyspnea and air trapping. Chest 1999; 115: 1207-1210.

18 Yap E, Low I. Bronchoscopic transbronchial cryobiopsy diagnosis of recurrent diffuse large B-cell lymphoma in the lung: a promising new tool? J Bronchology Interv Pulmonol 2017; 24: e22-e23.

19 Schiavo D, Batzlaff C, Maldonado F. Pulmonary parenchymal lymphoma diagnosed by bronchoscopic cryoprobe lung biopsy. J Bronchology Interv Pulmonol 2016; 23: 174-176. 